\title{
EXTREMA OF RANDOM ALGEBRAIC POLYNOMIALS WITH NON-IDENTICALLY DISTRIBUTED NORMAL COEFFICIENTS
}

\author{
K. FARAHMAND and A. GRIGORASH
}

(Received 1 June 1999; revised 13 September 2000)

\author{
Communicated by V. Stefanov
}

\begin{abstract}
An asymptotic estimate is derived for the expected number of extrema of a polynomial $a_{0}+a_{1}\left(\begin{array}{l}n \\ 1\end{array}\right)^{1 / 2} x+$ $a_{2}\left(\begin{array}{l}n \\ 2\end{array}\right)^{1 / 2} x^{2}+\cdots+a_{n}\left(\begin{array}{l}n \\ n\end{array}\right)^{1 / 2} x^{n}$ whose independent normal coefficients possess non-equal non-zero mean values. A result is presented that generalizes in terms of normal processes the analytical device used for construction of similar asymptotic estimates for random polynomials with normal coefficients.

2000 Mathematics subject classification: primary 60H99; secondary 60G15.

Keywords and phrases: number of real zeros, real roots, number of extrema, random algebraic polynomials, normal stochastic processes, asymptotic estimate.
\end{abstract}

\section{Introduction}

Let $\xi(t)$ be a real normal (Gaussian) stochastic process and $N(0,1)$ the number of the real zeros of $\xi(t)$ in the interval $0 \leq t \leq 1$. Denote $m(t)=E[\xi(t)]$ its mean function, $r(t, s)=\operatorname{cov}[\xi(t), \xi(s)]$ its covariance function, and

$$
r_{11}(t, s)=\frac{\partial^{2} r(t, s)}{\partial t \partial s}
$$

its mixed second order derivative.

There are many known results concerning the expected value $E N(0,1)$. These works are mainly aimed at relaxing the assumptions set on $\xi(t)$ in Theorem 1 below and obtaining the result for a more wide class of stochastic processes, see for example $[10,11]$. In particular, Theorem 1 remains true if the condition of continuity of

The work of A. Grigorash was supported by the Award \#98042004 from the ORS Awards Scheme and a scholarship of the Vice-Chancellor of the University of Ulster.

(C) 2001 Australian Mathematical Society 0263-6115/2001 \$A2.00+0.00 
the sample functions of $\xi(t)$ is replaced by the condition that $\xi(t)$ is separable [9]. However, Cramerr and Leadbetter, reviewing this one and other earlier results in the fundamental book [3], used the argument due to Belyaev [1] to demonstrate that the continuity conditions are not very restrictive. Here we restate Theorem 1 as it was in [3, page 285]:

THEOREM 1. If the process $\xi(t)$ possesses continuous sample functions with probability one; its covariance function $r(t, s)$, mean function $m(t)$ and its derivative $m^{\prime}(t)$ are continuous; the function $r_{11}(t, t)$ is continuous at all diagonal points $(t, t)$; the joint distribution for $\xi(t)$ and the quadratic mean derivative $\xi^{\prime}(t)$ is nonsingular for each $t$ such that $r(t, t)>0$ and $r(t, t) r_{11}(t, t)>r_{01}^{2}(\star t)$, then the expected number of real zeros of $\xi(t)$ within $[0,1]$ is

$$
E N(0,1)=\int_{0}^{1} \frac{\sqrt{\left(1-\rho^{2}\right) r_{11}(t, t)}}{\sigma} \varphi\left(\frac{m}{\sigma}\right)\{2 \varphi(\eta)+\eta(2 \Phi(\eta)-1)\} d t,
$$

where

$$
\begin{aligned}
\sigma^{2} & =r(t, t), \\
\rho & =\frac{\operatorname{cov}\left[\xi(t), \xi^{\prime}(t)\right]}{\sigma \operatorname{var}\left[\xi^{\prime}(t)\right]}=\frac{r_{01}(t, t)}{\sigma \sqrt{r_{11}(t, t)}}=\frac{1}{\sigma \sqrt{r_{11}(t, t)}}\left[\frac{\partial r(t, s)}{\partial s}\right]_{s=t}, \\
\eta & =\frac{m^{\prime}(t)-\rho m(t) \sqrt{r_{11}(t, t)} / \sigma}{\sqrt{\left(1-\rho^{2}\right) r_{11}(t, t)}},
\end{aligned}
$$

and $\varphi(t)$ and $\Phi(t)$ denote the standard normal density and the standard normal distribution function.

Formula (1.1) is used in a number of other works including random polynomials to derive asymptotic estimates for the expected number of their real zeros, extrema, or level crossings, as the degree of the polynomial increases without bound (for example, see [4, 8, 7], [2, pages 87 and 108], and [6, parts 3-6]). These works, differing in detail, generally employ the following method in order to simplify the calculations. First, $E N(0,1)$ is represented by a sum of two terms,

$$
E N(0,1)=I_{1}(0,1)+I_{2}(0,1),
$$

where

$$
\begin{aligned}
& I_{1}(0,1)=\frac{1}{\pi} \int_{0}^{1} \frac{\sqrt{\left(1-\rho^{2}\right) r_{11}(t, t)}}{\sigma} \exp \left(-\frac{m^{2}}{2 \sigma^{2}}-\frac{\eta^{2}}{2}\right) d t \\
& I_{2}(0,1)=\frac{\sqrt{2}}{\pi} \int_{0}^{1} \frac{\sqrt{\left(1-\rho^{2}\right) r_{11}(t, t)}}{\sigma} \eta \exp \left(-\frac{m^{2}}{2 \sigma^{2}}\right) \operatorname{erf}\left(\frac{\eta}{\sqrt{2}}\right) d t
\end{aligned}
$$


and

$$
\operatorname{erf}(t)=\int_{0}^{t} \exp \left(-x^{2}\right) d x
$$

Hence the original double integral in formula (1.1) (the internal integration implicated by $\Phi(t))$ is decomposed into a sum of a single integral (1.5) and a double integral (1.6). Now if the value of (1.5) becomes large with the increase of $n$, then the next step of the method is an attempt to prove that the value of (1.6) is bounded by a relatively small value. In this case $I_{2}$ does not affect the final asymptotic relation, and thus the original double integration is reduced to a single integration.

Traditionally, in order to prove the boundedness of (1.6), specific features of a particular problem setup were utilized, which led to rather lengthy estimations $[12,6]$. However, two recent works [8] and [7] introduced an analytical device allowing a uniformly simple proof of the boundedness of (1.6) for many cases. The following theorem generalizes this technique for a class of random processes which is indeed of more general nature than random polynomials.

THEOREM 2. If the process $\xi(t)$ satisfies the conditions of Theorem 1, and the function $|m / \sigma|$ has finite limits or increases indefinitely as $t$ tends to 0 and as $t$ tends to 1 , then

$$
E N(0,1)=I_{1}(0,1)+O(1)
$$

where $I_{1}(0,1)$ is defined by $(1.5)$.

NOTE. Both theorems were formulated for the interval $[0,1]$. However the proof of (1.1) in [3], as well as the proof of Theorem 2 in Section 2 make it obvious that the corresponding formulae for the mean number of zeros of $\xi(t)$ in a wider interval $[0, T]$ are obtained simply by altering the upper integration limit to $T$. The formula for the expected number $E N_{K}$ of times when the process $\xi(t)$ crosses a continuously differentiable curve $K(t)$ alsdresults from (1.1) where it is sufficient to replace $m(t)$ by $m(t)-K(t)$ and $m^{\prime}(t)$ by $m^{\prime}(t)-K^{\prime}(t)$. Again, the same applies to the statement of Theorem 2.

As mentioned above, Theorem 2, of interest in its own right, can be fruitfully used in the theory of random polynomials. To this end we consider the random algebraic polynomial

$$
P(x) \equiv P_{n}(x, \omega)=\sum_{j=0}^{n} a_{j}(\omega)\left(\begin{array}{l}
n \\
j
\end{array}\right)^{1 / 2} x^{j},
$$


defined on a real axis $x \in \mathbb{R}$, with random coefficients $\left\{a_{j}(\omega)\right\}_{j=0}^{n}$ defined in a fixed probability space $(\Omega, \mathscr{A}, P r)$. Motivated by its physical applications, see [5], in recent years several papers studied behaviour of this type of random polynomials in cases when the coefficients are standard normal variables. Edelman and Kostlan [5] obtained a formula for the expected number of real zeros, Farahmand [6, page 51] derived asymptotic estimates for the expected number of level crossings and extrema. There are other types of random algebraic polynomials which are intensively studied, for example see [13], and are reviewed in [6].

We assume that $P(x)$ has independent normal random coefficients with non-zero mean values. Within this range of the coefficients of $P(x)$ in (1.8) the asymptotic estimate of the expected number of level crossings was obtained in [7]. In Section 3 of the present paper we prove the following statement.

THEOREM 3. If the coefficients $a_{j}(\omega)$ of $P(x)$ in (1.8) are independent normally distributed random variables with variance $\operatorname{var}\left[a_{j}(\omega)\right]=\sigma^{2}$ and expected value $E\left[a_{j}(\omega)\right]=\mu\left(\begin{array}{l}n \\ j\end{array}\right)^{1 / 2}, \mu \neq 0$, then for all sufficiently large $n$ the expected number of extrema of this polynomial satisfies

$$
E M(-\infty, 0) \sim \sqrt{n-1} / 2, \quad E M(0, \infty) \sim O(1) .
$$

\section{Proof of Theorem 2}

With notation established in Section 1 the proof of Theorem 2 is reduced to proving that $I_{2}(0,1)=O(1)$, where $I_{2}$ is defined by (1.6).

Let $u=m / \sigma$, where $\sigma$ is defined by (1.2). Then

$$
d u=\left(\frac{m^{\prime}}{\sigma}-\frac{m}{\sigma^{2}} \frac{d \sigma}{d t}\right) d t .
$$

By theorem's conditions, $m(t)$ is differentiable, hence the following equations hold:

$$
\begin{aligned}
& \frac{\partial r(t, s)}{\partial t}=\operatorname{cov}\left[\xi^{\prime}(t), \xi(s)\right] \equiv r_{10}(t, s), \\
& \frac{\partial r(t, s)}{\partial s}=\operatorname{cov}\left[\xi(t), \xi^{\prime}(s)\right] \equiv r_{01}(t, s) .
\end{aligned}
$$

By covariance properties, $r_{10}(t, t)=r_{01}(t, t)$

Since

$$
\frac{d \sigma^{2}}{d t}=\left[\frac{\partial r(t, s)}{\partial s}\right]_{s=t}+\left[\frac{\partial r(t, s)}{\partial t}\right]_{s=t}=2 r_{01}(t, t) .
$$

$$
\frac{d \sigma}{d t}=\frac{1}{2 \sigma} \frac{d \sigma^{2}}{d t}
$$


(2.1) and (2.2) after expansion of $\rho$ and $\eta$ by definitions (1.3) and (1.4) produce

$$
\frac{\sqrt{\left(1-\rho^{2}\right) r_{11}(t, t)}}{\sigma} \eta=\frac{d u}{d t} .
$$

Then the integration variable in (1.6) can be changed from $t$ to $u$, such that

$$
I_{2}(0,1)=\frac{\sqrt{2}}{\pi} \int_{u^{-}}^{u^{+}} \exp \left(-\frac{u^{2}}{2}\right) \operatorname{erf}(\eta(t(u))) d u,
$$

where

$$
u^{+}=\lim _{t \rightarrow 1} \frac{m}{\sigma} \quad \text { and } \quad u^{-}=\lim _{t \rightarrow 0} \frac{m}{\sigma} .
$$

The limits of the integration exist as finite numbers or infinite values by conditions of the theorem. From (1.7), $\operatorname{erf}(t) \leq \sqrt{\pi} / 2$. Therefore

$$
\begin{aligned}
I_{2}(0,1) & \leq \frac{1}{\sqrt{2 \pi}} \int_{u^{-}}^{u^{+}} \exp \left(-\frac{u^{2}}{2}\right) d u=\Phi\left(\lim _{t \rightarrow 1} \frac{m}{\sigma}\right)-\Phi\left(\lim _{t \rightarrow 0} \frac{m}{\sigma}\right) \\
& \leq \Phi(+\infty)-\Phi(-\infty)=1 .
\end{aligned}
$$

Thus $I_{2}(0,1)=O(1)$ with implied constant 1 . The theorem is proved.

\section{Random algebraic polynomials}

In this section we prove Theorem 3. From [3] and [6] it follows that the probability that $P(x)$ defined in (1.8) has a point of horizontal inflexion is zero. Then the expected number $E M(\alpha, \beta)$ of extrema of $P(x)$ in an interval $[\alpha, \beta]$ is the expected number of real zeros in $[\alpha, \beta]$ of its derivative

$$
P^{\prime}(x) \equiv P_{n}^{\prime}(x, \omega)=\sum_{j=1}^{n} j a_{j}(\omega)\left(\begin{array}{l}
n \\
j
\end{array}\right)^{1 / 2} x^{j-1} .
$$

Denote

$$
\begin{aligned}
m_{1} & =E\left[P_{n}^{\prime}(x, \omega)\right] \doteq E\left[P^{\prime}(x)\right]=\mu n(1+x)^{n-1}, \\
m_{2} & =E\left[P^{\prime \prime}(x)\right]=\mu n(n-1)(1+x)^{n-2}, \\
A^{2} & =\operatorname{var}\left[P^{\prime}(x)\right]=\sigma^{2} n\left(1+n x^{2}\right)\left(1+x^{2}\right)^{n-2}, \\
B^{2} & =\operatorname{var}\left[P^{\prime \prime}(x)\right]=\sigma^{2} n(n-1)\left(2+(n-1) x^{2}\left(4+n x^{2}\right)\right)\left(1+x^{2}\right)^{n-4}, \\
C & =\operatorname{cov}\left[P^{\prime}(x), P^{\prime \prime}(x)\right]=\sigma^{2} n(n-1) x\left(2+n x^{2}\right)\left(1+x^{2}\right)^{n-3}, \\
\Delta^{2} & =A^{2} B^{2}-C^{2}=\sigma^{4} n^{2}(n-1)\left(1+x^{2}\right)^{2 n-6}\left(2+2 n x^{2}+n(n-1) x^{4}\right) .
\end{aligned}
$$

Obviously, (3.1) satisfies the conditions of Theorem 1. Note that formula (1.1) holds in this case not only for a finite interval $[0, T]$ but also for the whole axis $(-\infty, \infty)$. 
The existence of the improper integral in the latter case is secured by the fact that the continuous integrand in (1.1) is bounded, since the number of zeros of polynomial (3.1) is finite.

The value $m(t) / \sigma(t)$ from Theorem 2 formulation is denoted in notation (3.2) by $m_{1}(x) / A(x)$. Both limits

$$
\lim _{x \rightarrow \infty} \frac{m_{1}(x)}{A(x)}=\frac{\mu}{\sigma} ; \quad \lim _{x \rightarrow-\infty} \frac{m_{1}(x)}{A(x)}=(-1)^{n-1} \frac{\mu}{\sigma}
$$

exist, so Theorem 2 is applicable and yields

$$
E M(-\infty, \infty)=\int_{-\infty}^{\infty} \frac{\Delta}{\pi A^{2}} \exp \left(-\frac{m_{1}^{2}}{2 A^{2}}-\frac{\left(A^{2} m_{2}-C m_{1}\right)^{2}}{2 A^{2} \Delta^{2}}\right) d x+O(1)
$$

Thus the task of asymptotic estimation of $E M(-\infty, \infty)$ is reduced to the evaluation of the integral in (3.3). Denote

$$
\begin{aligned}
\mathscr{T}(x)= & \frac{m_{1}^{2}}{2 A^{2}}+\left(\frac{A^{2} m_{2}-C m_{1}}{\sqrt{2} A \Delta}\right)^{2} \\
= & \frac{\mu^{2} n(1+x)^{2 n-2}}{2 \sigma^{2}\left(1+n x^{2}\right)\left(1+x^{2}\right)^{n-2}} \\
& +\frac{\mu^{2} n(n-1)(1+x)^{2(n-2)}\left[\left(1+n x^{2}\right)\left(1+x^{2}\right)-x(1+x)\left(2+n x^{2}\right)\right]^{2}}{2 \sigma^{2}\left(1+n x^{2}\right)\left(1+x^{2}\right)^{n-2}\left(2+2 n x^{2}+n(n-1) x^{4}\right)} .
\end{aligned}
$$

Then

$$
E M(-\infty, \infty)=I_{1}(-\infty, \infty)+O(1)
$$

where

$$
I_{1}(-\infty, \infty)=\int_{-\infty}^{\infty} \frac{\Delta}{\pi A^{2}} \exp (\mathscr{T}(x)) d x .
$$

The estimation of $I_{1}(-\infty, \infty)$ is carried out for two groups of intervals: (i) $[-1,1]$ and (ii) $(-\infty, 1) \cup(1, \infty)$.

(i) $x \in[-1,1]$.

Consider first $-1 \leq x<0$. Denote $y=-x$, then

$$
\begin{aligned}
\mathscr{T}(y)= & \frac{\mu^{2} n(1-y)^{2 n-2}}{2 \sigma^{2}\left(1+n y^{2}\right)\left(1+y^{2}\right)^{n-2}} \\
& +\frac{\mu^{2} n(n-1)(1-y)^{2(n-2)}\left[1+2 y+(n-1) y^{2}+n y^{3}\right]^{2}}{2 \sigma^{2}\left(1+n y^{2}\right)\left(1+y^{2}\right)^{n-2}\left(2+2 n y^{2}+\left(n^{2}-n\right) y^{4}\right)} .
\end{aligned}
$$

Choose a small constant

$$
\varepsilon=\tan \left(n^{-3 / 4}\right)
$$


and split the interval $[-1,0)$ in two, such that $I_{1}(-1,0)=I_{1}(-1,-\varepsilon)+I_{1}(-\varepsilon, 0)$. Obviously, the interval $x \in[-1,-\varepsilon)$ corresponds to $y \in(\varepsilon, 1]$; the interval $x \in$ $[-\varepsilon, 0)$ corresponds to $y \in(0, \varepsilon]$. Within $\varepsilon<y \leq 1(-1 \leq x<\varepsilon)$

$$
\begin{aligned}
\mathscr{T} & <\frac{\mu^{2}}{2 \sigma^{2}} \frac{n(1-\varepsilon)^{2 n-4}}{\left(1+n \varepsilon^{2}\right)\left(1+\varepsilon^{2}\right)^{n-2}}\left((1-\varepsilon)^{2}+\frac{(n-1)[2 n-2]^{2}}{2+2 n \varepsilon^{2}+\left(n^{2}-n\right) \varepsilon^{4}}\right) \\
& <\frac{\mu^{2}}{2 \sigma^{2}} \frac{n(1-\varepsilon)^{2 n-4}}{\left(1+\varepsilon^{2}\right)^{n-2}}\left(1+2 n^{3}\right)
\end{aligned}
$$

and

$$
\begin{aligned}
\frac{(1-\varepsilon)^{2 n-4}}{\left(1+\varepsilon^{2}\right)^{n-2}} & =\left(1-\frac{2 \varepsilon}{1+\varepsilon^{2}}\right)^{n-2} \simeq\left(1-\frac{2}{n^{3 / 4}}\right)^{n-2} \\
& \simeq \exp \left(-\frac{2(n-2)}{n^{3 / 4}}\right)=O\left(\exp \left(-n^{1 / 4}\right)\right) .
\end{aligned}
$$

Clearly, the right-hand side in inequality (3.6) is $O\left(n^{4} e^{-n^{1 / 4}}\right)=O\left(e^{-n^{1 / 4}+\ln n}\right) \rightarrow 0$ as $n \rightarrow \infty$. Therefore $\mathscr{T}(y) \rightarrow 0$ as $n \rightarrow \infty$, and

$$
I_{1}(-1,-\varepsilon) \simeq \frac{1}{\pi} \int_{\varepsilon}^{1} \frac{\Delta}{A^{2}} d x .
$$

Note that

$$
\lim _{n \rightarrow \infty} \frac{\Delta}{A^{2} \sqrt{n-1}}=\frac{1}{x^{2}+1} .
$$

Then the dominated convergence theorem for Lebesgue integrals shows that

$$
\int_{\varepsilon}^{1} \frac{\Delta}{A^{2} \sqrt{n-1}} d x \simeq \int_{\varepsilon}^{1} \frac{1}{x^{2}+1} d x=\frac{\pi}{4}-n^{-3 / 4} .
$$

From (3.8) and (3.9) with $n \rightarrow \infty$ we obtain $I_{1}(-1,-\varepsilon) \simeq \sqrt{n-1} / 4+O\left(n^{-1 / 4}\right)$.

Within $0<y \leq \varepsilon$, that is, $-\varepsilon \leq x<0$, we note that for sufficiently large $n$,

$$
\begin{aligned}
0 \leq I_{1}(-\varepsilon, 0) & \leq \frac{1}{\pi} \int_{0}^{\varepsilon} \frac{\Delta}{A^{2}} d x \simeq \frac{\sqrt{n-1}}{\pi} \int_{0}^{\varepsilon} \frac{1}{1+x^{2}} d x \\
& =\frac{\sqrt{n-1}}{\pi n^{3 / 4}} \rightarrow 0 \text { as } n \rightarrow \infty .
\end{aligned}
$$

Thus,

$$
I_{1}(-1,0) \simeq \frac{\sqrt{n-1}}{4}+O\left(n^{-1 / 4}\right) \text { as } n \rightarrow \infty .
$$

Now consider $0 \leq x \leq 1$. From (3.4), ignoring the second term produces an inequality

$$
\mathscr{T}(x) \geq \frac{\mu^{2} n\left(1+x^{2}\right)}{2 \sigma^{2}\left(1+n x^{2}\right)}\left(\frac{(1+x)^{2}}{1+x^{2}}\right)^{n-1} .
$$


Within the subinterval $\varepsilon \leq x \leq 1$, this inequality can be strengthened as

$$
\mathscr{T}(x)>\frac{\mu^{2} n\left(1+\varepsilon^{2}\right)}{8 \sigma^{2}(1+n)}(1+\varepsilon)^{n-1}=L(n), \quad \text { say. }
$$

Hence

$$
I_{1}(\varepsilon, 1) \leq \frac{\sqrt{n-1}}{\pi} \exp (-L(n)) \int_{\varepsilon}^{1} \frac{d x}{1+x^{2}} .
$$

By the choice of $\varepsilon$ in (3.5) it is easy to verify that $L(n)$ increases without bound as $n \rightarrow \infty$. This makes the right-hand side of the last inequality tend to zero. Recalling that $l_{1}$ by its definition is nonnegative, we conclude that

Within $0 \leq x<\varepsilon$

$$
\lim _{n \rightarrow \infty} I_{1}(\varepsilon, 1)=0 \text {. }
$$

$$
\mathscr{T}(x)>\frac{\mu^{2} n}{8 \sigma^{2}(1+n)},
$$

and Thus

$$
I_{1}(0, \varepsilon) \leq \frac{\sqrt{n-1}}{\pi} \exp \left(-\frac{\mu^{2} n}{8 \sigma^{2}(1+n)}\right) \int_{0}^{\varepsilon} \frac{d x}{1+x^{2}} \rightarrow 0 \text { as } n \rightarrow \infty .
$$

$$
\lim _{n \rightarrow \infty} I_{1}(0,1)=0
$$

(ii) $x \in(-\infty, 1) \cup(1, \infty)$.

The substitution of $z=1 / x$ implies $z \in(-1,0) \cup(0,1)$. Within $0<z<1$ utilising inequalities similar to (3.12), (3.13), and (3.14) leads to the conclusion that

$$
I_{1}(1, \infty) \rightarrow 0 \text { as } n \rightarrow \infty .
$$

Consider $-1<z<0$. After another change of variable $y=-z$, establishment of relations analogous to (3.6), (3.7), (3.8), and (3.9) yields

$$
I_{1}(-1 / \varepsilon,-1) \simeq \sqrt{n-1} / 4+O\left(n^{-1 / 4}\right)
$$

for the interval $\varepsilon \leq y<1$ (equivalent to $-1<z \leq-\varepsilon$, to $-1 / \varepsilon \leq x<-1$ ). Treatment of the interval $0 \leq y<\varepsilon(-\varepsilon<z<0)$ leads to an outcome analogous to (3.10):

$$
I_{1}(-\infty,-1 / \varepsilon) \rightarrow 0 \text { as } n \rightarrow \infty
$$

Therefore,

$$
I_{1}(-\infty,-1) \simeq \sqrt{n-1} / 4+O\left(n^{-1 / 4}\right) .
$$

Putting together (3.11), (3.15), (3.16), and (3.17) produces the desired result. 


\title{
References
}

[1] Yu. K. Belayev, 'Continuity and Hölder's conditions for sample functions of stationary Gaussian processes', Proc. 4th Berkeley Sympos. on Math. Statist. and Prob. II (Univ. California Press, Berkeley, 1961), 23-33.

[2] A. T. Bharucha-Reid and M. Sambandham, Random polynomials (Academic Press, New York, 1986).

[3] H. Cramér and M. R. Leadbetter, Stationary and related stochastic processes (Wiley, New York, 1967).

[4] M. Das, 'The average number of maxima of a random algebraic curve', Proc. Cambridge Philos. Soc. 65 (1969), 741-753.

[5] A. Edelman and E. Kostlan, 'How many zeros of a random polynomial are real?', Bull. Amer. Math. Soc. 32 (1995), 1-37.

[6] K. Farahmand, Topics in random polynomials (Addison Wesley Longman, London, 1998).

[7] K. Farahmand and A. Grigorash, 'Level crossings of random algebraic polynomials with nonidentically distributed normal coefficients', Int. J. Appl. Math. 2 (2000), 213-221.

[8] K. Farahmand and P. Hannigan, 'The expected number of local maxima of a random algebraic polynomial', J. Theoret. Probab. 10 (1997), 991-1002.

[9] M. R. Leadbetter, 'On crossings of levels and curves by a wide class of stochastic processes', Ann. Math. Statist. 37 (1966), 260-267.

[10] M. B. Marcus, 'Level crossings of a stochastic process with absolutely continuous sample paths', Ann. Probab. 5 (1977), 52-71.

[11] Z. Michna and I. Rychlik, 'The expected number of level crossings for certain symmetric $\alpha$-stable processes', Comm. Statist. Stochastic Models 11 (1995), 1-19.

[12] M. Sambandham, 'On random trigonometric polynomial', Indian J. Pure Appl. Math. 7 (1976), 841-849.

[13] J. E. Wilkins, 'An asymptotic expansion for the expected number of real zeros of a random polynomial', Proc. Amer. Math. Soc. 103 (1988), 1249-1258.

\author{
Department of Mathematics, \\ University of Ulster at Jordanstown, \\ Co. Antrim, BT37 0QB, \\ United Kingdom \\ e-mail: K.Farahmand@ulst.ac.uk, AV.Grigorash@ulst.ac.uk
}


